\title{
Association between polymorphisms in CHRNA3 and PHACTR2 gene and environment and NSCLC risk in Chinese population
}

\author{
Guangyuan Lou', Yongjun Zhang ${ }^{2 凶}$, Wenlong $\mathrm{Bao}^{2}$ and Dehou Deng ${ }^{2}$ \\ 'Department of Chemotherapy, Zhejiang Cancer Hospital, Hangzhou, China; ${ }^{2}$ Department of Integration of Traditional Chinese and Western \\ Medicine, Zhejiang Cancer Hospital, Hangzhou, China
}

\begin{abstract}
Aims. This study aimed to investigate CHRNA3 (rs8040868) and PHACTR2 (rs9390123) single-nucleotide polymorphisms (SNPs) for association with non-small-cell lung cancer (NSCLC) risk in a Chinese population, and whether the environment affects the genetic polymorphisms. Methods. This case and control study included 500 NSCLC patients and 500 age-matched healthy controls. CHRNA3 (rs8040868) and PHACTR2 (rs9390123) SNPs were genotyped and associated for NSCLC risk by computing the odds ratio and $95 \%$ confidence interval from multivariate unconditional logistic regression analyses with adjustment of age. Results. The minor allele frequency (MAF) of CHRNA3 (rs8040868) and PHACTR2 (rs9390123) was 0.350 (C) and 0.397 (C), respectively. The frequencies of genotype and allele in CHRNA3 (rs8040868) and PHACTR2 (rs9390123) were not significantly different between the cases and controls, or between either of the subgroups. Conclusion. Although rs8040868 and rs9390123 SNPs are not associated with NSCLC risk in Chinese population, the results strongly suggest that geographical agents interact with human genetic polymorphism independent of ethnic background.
\end{abstract}

Key words: Non-small cell lung cancer, phosphatase and actin regulator 2 (PHACTR2), cholinergic receptor, nicotinic, alpha 3 (CHRNA3), single-nucleotide polymorphism

Received: 21 March, 2014; revised: 03 July, 2014; accepted: 05 November, 2014; available on-line: 14 November, 2014

\section{INTRODUCTION}

Lung cancer is the most commonly diagnosed cancer and the leading cause of cancer death in males. Futhermore, it is the fourth most commonly diagnosed cancer and the second leading cause of cancer death among females (Jemal et al., 2011). Non-small cell lung cancer (NSCLC) is the most common histological subtype and accounts for approximately $85 \%$ of all lung cancer cases (Molina et al., 2008). Smoking accounts for $80 \%$ of the worldwide lung cancer burden in males and at least 50\% of the burden in females (Ezzati et al., 2005; Ezzati et al., 2003). Other known risk factors for lung cancer include exposure to several occupational and geographical carcinogens such as asbestos, arsenic, radon, and polycyclic aromatic hydrocarbons (Spitz et al., 2006). However, not all people who were exposed to those risk factors develop lung cancer. Thus, it is biologically conceivable that ethnic background and host genetic susceptibility are important factors in lung cancer development.
Recently, a genome-wide gene association study reported that, among U.S. residents of European ancestry, the polymorphism rs9390123 in phosphatase and actin regulator 2 (PHACTR2) was associated with lung cancer (Wang et al., 2013). Thus, in this study, we genotyped PHACTR2 rs9390123 Single Nucleotide Polymorphism (SNP) in 500 NSCLC patients and 500 age-matched healthy controls, and then associated for NSCLC risk in a Chinese population by computing the odds ratio, and $95 \%$ confidence interval from multivariate unconditional logistic regression analyses with adjustment of age. We also evaluated the association of rs8040868 SNPs in the cholinergic receptor, nicotinic, alpha 3 (CHRNA3) gene, which was associated with lung cancer risk among Caucasians (Chikova et al., 2012).

\section{MATERIALS AND METHODS}

Study population. A total of 500 NSCLC patients and 500 unrelated healthy controls were recruited from The Zhejiang Cancer Hospital, Hangzhou, China between March 2011 and April 2012. All cases and controls were of Chinese Han origin and lived in the same geographic region (Zhejing Province, China). Exclusion criteria included a history of previous primary cancer other than lung cancer. The controls were free of lungrelated disease to avoid any probable interference from overlapping genes. The control subjects were related to age-matched patients. A regular smoker was defined as someone who had smoked more than one pack/year (py); and a current smoker or former smoker was defined as a regular smoker who still smoked in the year of the interview or in the previous year (Pesch et al., 2012). This study was approved by the Ethics Committee of Zhejiang Cancer Hospital, and all of the studied subjects provided an informed consent.

SNP selection and genotyping. PHACTR2 rs9390123 and CHRNA3 rs8040868 were selected based on Wang et al. (2013) and Chikova et al., (2012). For genotyping of SNPs, genomic DNA was extracted from whole blood using the AxyPrep Blood Genomic DNA Miniprep kit (Axygen Biosciences, Union City, CA) and subjected to genotyping of SNPs with the SEQUENOM MassARRAY matrix-assisted laser desorption ionization-

e-mail: zhangyj@zjcc.org.cn

Abbreviations: ADC, adenocarcinoma; CHRNA3, cholinergic receptor, nicotinic, alpha 3; Cl, confidence interval; HWE, Hardy-Weinberg equilibrium; MAF, minor allele frequency; NSCLC: non-smallcell lung cancer; OR: odds ratio; PHACTR2: cholinergic receptor, nicotinic, alpha 3; SCC: squamous-cell carcinoma; SNPs: single-nucleotide polymorphisms 
time of flight mass spectrometry platform (Sequenom, San Diego, CA). Primers for the polymerase chain reaction and single base extension were designed using the Assay Designer's software version 3.0 (Sequenom) and synthesized by Sangon Biotech (Shanghai, China): CHRNA3 rs8040868 primers: 1st, 5'-ACGTTGGATGGATTACAATGAGATCATCCG-3'; 2nd, 5'-ACGTTGGATGTGGACACCTCGAAATGGATG-3'; and extension, 5'-GGTCAGACACGTTGGGC-3'. PHAC TR2 rs9390123 primers: 1st, 5'-ACGTTGGATGGCAGGATCTCTGGAGATTTC-3'; 2nd, 5'-ACGTTGGATGACATAATGGAGGTGGACAGC-3'; and extension, 5'-GGTGGACAGCTAGGTTA-3'.

Statistical analysis. All statistical calculations were performed using SPSS 13.0 for Windows (SPSS Inc., Chicago, IL). Hardy-Weinberg equilibrium (HWE) testing was carried out for all SNPs by using the $\chi^{2}$ test, and $P<0.001$ was considered significantly different between case and control. The $\chi^{2}$ test was also used to assess frequencies of the selected allele and genotype between the cases and controls. The association between SNPs and NSCLC risk was evaluated by computing the odds ratio $(\mathrm{OR})$ and $95 \%$ confidence interval (CI) from multivariate unconditional logistic regression analysis.

\section{RESULTS}

\section{Description and clinical characteristics of the study population}

A total of 500 patients (350 males and 150 females) and 500 healthy controls (259 males, 240 females, and 1 unknown) were enrolled in this study. For NSCLC patients, 331 had adenocarcinoma and 169 had squamous-cell carcinoma (SCC); 280 male and 21 female patients were smokers or former smokers, while 189 male and 14 females among the controls were smokers or former smokers. The studied population was within the HWE for CHRNA3 rs8040868 and PHACTR2 rs9390123, and the $P$ values were 0.005 and 0.66 , respectively.

\section{Frequencies of CHRNA3 rs8040868 and PHACTR2 rs9390123 polymorphisms between cases and controls}

The allele frequencies of CHRNA3 rs8040868 were $64.1 \%(\mathrm{~T})$ and $35.9 \%(\mathrm{C}), 62.8 \%(\mathrm{~T})$ and $37.2 \%(\mathrm{C})$, $66.6 \%(\mathrm{~T})$ and $33.4 \%(\mathrm{C})$, and $65.0 \%(\mathrm{~T})$ and $35.0 \%$ (C) in NSCLC patients, ADC patients, SCC patients, and controls, respectively; while the allele frequencies of PHACTR2 rs 9390123 were $61.8 \%(\mathrm{~T})$ and $38.2 \%(\mathrm{C})$, $62.5 \%(\mathrm{~T})$ and $37.5 \%(\mathrm{C})), 60.4 \%(\mathrm{~T})$ and $39.6 \%(\mathrm{C})$, and $60.3 \%(\mathrm{~T})$ and $39.7 \%(\mathrm{C})$ in NSCLC patients, ADC patients, SCC patients, and controls, respectively. There were no statistically significant differences in terms of the allele frequencies of these two SNPs between controls and patients with NSCLC, ADC or SCC. We also stratified the data by gender and found that there were no statistical differences between cases and controls ( $\mathrm{Ta}-$ ble 1).

The genotype distribution data of the cases and controls are shown in Table 2 (between genotype frequencies of CHRNA3 rs8040868 and PHACTR2 rs9390123). There were no statistically significant differences between NSCLCs and controls, and NSCLC subgroups and control subgroups. When analyzing the association between genotypes and the risk of NSCLC, logistic regression analysis revealed that polymorphisms of CHR- 


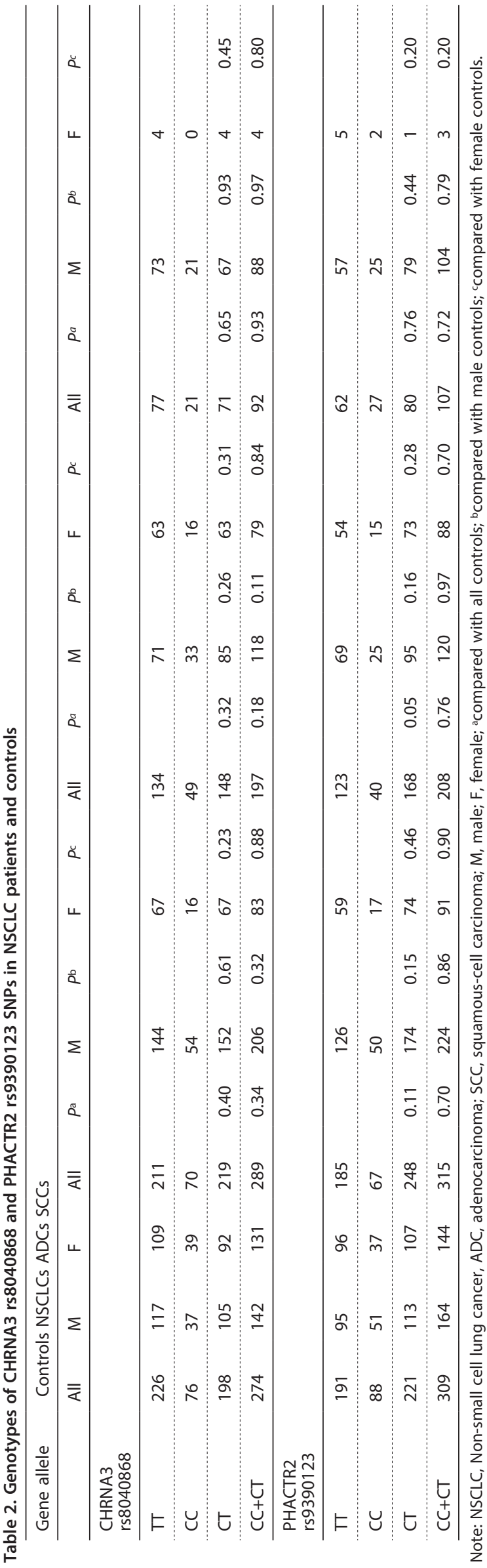

NA3 rs8040868 and PHACTR2 rs9390123 were not associated with NSCLC development.

\section{DISCUSSIONS}

In this current study, we investigated CHRNA3 rs8040868 and PHACTR2 rs9390123 SNPs in 500 NSCLC patients and 500 age-matched healthy controls for association with NSCLC risk in a Chinese population. The data showed that there is no association between SNPs and NSCLC risk in Chinese and this is the first study in a Chinese population to show this pattern.

Sequence variants in CHRNA SNPs on chromosome 15 have been associated with increased (self-reported) cigarette dose and nicotine dependence (Saccone et al., 2007) and increased risk of lung cancer in smokers (Thorgeirsson et al., 2008; Le Marchand et al., 2008), whereas such association in nonsmokers was not observed (Le Marchand et al., 2008). CHRNA SNPs that conferred lung cancer susceptibility in a smoking-independent Japanese manner (Shiraishi et al., 2009) were associated with risk of familial lung cancer, whereas association of these SNPs with smoking status was not significant in Americans (Liu et al., 2008).

In contrast to CHRNA3, the associations between SNPs of PHACTR2 and lung cancer were sparsely researched. PHACTR2 is located on 6q24, and encodes the protein phosphatase and actin regulator 2 that belongs to the PHACTR family containing four members (PHACTR1-4), which are abundantly expressed in the nervous system (Allen et al., 2004). Even though little is known of the proteins' function, they are suggested to regulate protein phosphatase 1 and to bind to cytoplasmic actin. Rs9390123 is located in an intron in the PHACTR2 gene. Wang and coworkers (2013) was the first to report about the association between SNP of rs9390123 and lung cancer risk.

In this study, we found that CHRNA3 rs8040868 and PHACTR2 rs9390123 SNPs were not associated with NSCLC among Chinese males. It is important to note that, in our previous study, we found that CHRNA3 polymorphism was not associated with NSCLC among non-smoking Chinese (Li et al., 2013). In both mentioned studies, it was suggested that CHRNA3 SNPs were no associated with NSCLC risk in Southern Chinese. However, the present data were markedly discordant compared with that of previously published studies (Wang et al., 2013; Chikova et al., 2012). The reasons for this discrepancy are unknown, but ethnic background of such patient populations may play a role. In other words, the essentiality of racial diversities may account for the candidate genes for association with NSCLC. Since minor allele frequency (MAF) of SNPs varies significantly between populations, association based on these SNPs will be particularly sensitive to ethnic variability. In this study, the HapMap database showed a great variability in MAF of CHRNA3 rs8040868 (http:/ / www.ncbi.nlm.nih.gov/projects/SNP/snp_ref.cgi? rs= 8040868) and PHACTR2 rs9390123 (http://www.ncbi. nlm.nih.gov/ projects/SNP/snp_ref.cgi?rs=9390123) among different populations. For example, the MAF of rs8040868 was 0.305 among CHB (Han Chinese in Beijing, China), 0.353 among CHD (Chinese in Metropolitan Denver, Colorado), and 0.367 among ASW (African ancestry in Southwest USA); the MAF of rs9390123 was 0.500 among $\mathrm{CHB}, 0.446$ among $\mathrm{CHD}$, and 0.337 among ASW (African ancestry in Southwest USA). Our study, the MAF of CHRNA3 rs8040868 and PHACTR2 
rs9390123 was 0.350 and 0.397 , respectively, which was inconsistent with the HapMap database. It suggested that geographical agents have a potential for interacting with human genetic polymorphisms independent of ethnic background (our population was Han Chinese in Zhejiang, China), just like in our study (Zhang et al., 2012).

In summary, we found that SNPs of CHRNA3 rs8040868 and PHACTR2 rs9390123 were not associated with NSCLC risk among Chinese. Our data suggest that geographical agents as well as ethnic background may play an important role in genetic polymorphism development.

\section{Conflict of interest statement}

Author declared no conflicts of interest with regard to this work.

\section{Acknowledgements}

We appreciated Xiaohong $\mathrm{Xu}$ and Yejiang Bao for their kind help in control screening. We also thank Hailong Liu for his excellent technical support.

\section{REFERENCES}

Allen PB, Greenfield AT, Svenningsson P, Haspeslagh DC, Greengard P (2004) Phactrs 1-4: A family of protein phosphatase 1 and actin regulatory proteins. Proc Natl Acad Sci U S A 101: 7187-7192.

Chikova A, Bernard HU, Shchepotin IB, Grando SA (2012) New associations of the genetic polymorphisms in nicotinic receptor genes with the risk of lung cancer. Life Sci 91: 1103-1108.

Ezzati M, Henley SJ, Lopez AD, Thun MJ (2005) Role of smoking in global and regional cancer epidemiology: current patterns and data needs. Int J Cancer 116: 963-971.

Ezzati M, Lopez AD (2003) Estimates of global mortality attributable to smoking in 2000. Lancet 362: 847-852.
Jemal A, Bray F, Center MM, Ferlay J, Ward E, Forman D (2011) Global cancer statistics. CA Cancer J Clin 61: 69-90.

Le Marchand L, Derby KS, Murphy SE, Hecht SS, Hatsukami D, Carmella SG et al (2008) Smokers with the CHRNA lung cancerassociated variants are exposed to higher levels of nicotine equivalents and a carcinogenic tobacco-specific nitrosamine. Cancer Res 68: 9137-9140.

Li Z, Bao S, Xu X, Bao Y, Zhang Y (2012) Polymorphisms of CHRNA5-CHRNA3-CHRNB4 Gene cluster and NSCLC risk in Chinese population. Transl Oncol 5: 448-452.

Liu P, Vikis HG, Wang D, Lu Y, Wang Y, Schwartz AG et al (2008) Familial aggregation of common sequence variants on 15q24-25.1 in lung cancer. J Natl Cancer Inst 100: 1326-1330.

Molina JR, Yang P, Cassivi SD, Schild SE, Adjei AA (2008) Non-small cell lung cancer: epidemiology, risk factors, treatment, and survivorship. Mayo Clin Proc 83: 584-594.

Pesch B, Kendzia B, Gustavsson P, Jöckel KH, Johnen G, Pohlabeln $\mathrm{H}$ et al (2012) Cigarette smoking and lung cancer--relative risk estimates for the major histological types from a pooled analysis of case-control studies. Int J Cance 131: 1210-1219.

Saccone SF, Hinrichs AL, Saccone NL, Chase GA, Konvicka K, Madden PA et al (2007) Cholinergic nicotinic receptor genes implicated in a nicotine dependence association study targeting 348 candidate genes with 3713 SNPs. Hum Mol Genet 16: 36-49.

Shiraishi K, Kohno T, Kunitoh H, Watanabe S, Goto K, Nishiwaki Y et al (2009) Contribution of nicotine acetylcholine receptor polymorphisms to lung cancer risk in a smoking-independent manner in the Japanese. Carcinogenesis 30: 65-70.

Spitz M, Wu X, Wilkinson A, Wei Q (2006) Cancer of the Lung. Oxford, UK: Oxford University Press.

Thorgeirsson TE, Geller F, Sulem P, Rafnar T, Wiste A, Magnusson $\mathrm{KP}$ et al (2008) A variant associated with nicotine dependence, lung cancer and peripheral arterial disease. Nature 452: 638-642.

Wang LE, Gorlova OY, Ying J, Qiao Y, Weng SF, Lee AT, et al (2013) Genome-wide association study reveals novel genetic determinants of DNA repair capacity in lung cancer. Cancer Res 73: 256-264.

Zhang Y, Gu C, Shi H, Zhang A, Kong X, Bao W et al (2012) Association between C3orf21, TP63 polymorphisms and environment and NSCLC in never-smoking Chinese population. Gene 497: 93-97. 